\title{
Lossy Compression
}

National Cancer Institute

\section{Source}

National Cancer Institute. Lossy Compression. NCI Thesaurus. Code C48366.

A data compression algorithm that reduces the amount of information in the data, rather than just the number of bits used to represent that information. 